\title{
LRS Bianchi Type-I Dark Energy Cosmological Models in General Scalar Tensor Theory of Gravitation
}

\author{
V. U. M. Rao and D. Neelima \\ Department of Applied Mathematics, Andhra University, Visakhapatnam 530003, India \\ Correspondence should be addressed to V. U. M. Rao; umrao57@hotmail.com
}

Received 19 April 2013; Accepted 29 May 2013

Academic Editors: H. Bushouse, M. Grewing, D. Kieda, V. Pierrard, K. P. Rauch, and W. W. Zeilinger

Copyright ( 2013 V. U. M. Rao and D. Neelima. This is an open access article distributed under the Creative Commons Attribution License, which permits unrestricted use, distribution, and reproduction in any medium, provided the original work is properly cited.

Locally rotationally symmetric (LRS) Bianchi type-I dark energy cosmological model with variable equation of state (EoS) parameter in (Nordtvedt 1970) general scalar tensor theory of gravitation with the help of a special case proposed by (Schwinger 1970 ) is obtained. It is observed that these anisotropic and isotropic dark energy cosmological models always represent an accelerated universe and are consistent with the recent observations of type-Ia supernovae. Some important features of the models, thus obtained, have been discussed.

\section{Introduction}

Nordtvedt [1] proposed a general class of scalar tensor gravitational theories in which the parameter $\omega$ of the Brans-Dicke (BD) theory is allowed to be an arbitrary (positive definite) function of the scalar field $(\omega \rightarrow \omega(\phi))$. Considering the static spherically symmetric solution for a point mass source, Nordtvedt [1] found a variety of experimental consequences of $\omega^{\prime} \neq 0$, including a contribution to the rate of precession of Mercury's perihelion.

Several investigations have been made in higher dimensional cosmology in the framework of different scalar tensor theories of gravitation. Barker [2], Ruban and Finkelstein [3], Banerjee and Santos $[4,5]$, and Shanti and Rao $[6,7]$ are some of the authors who have investigated several aspects of the Nordtvedt general scalar tensor theory in four dimensions. Rao and Sreedevi Kumari [8] have discussed a cosmological model with negative constant deceleration parameter in a general scalar tensor theory of gravitation. Rao et al. [9] have obtained the Kaluza-Klein radiating model in a general scalar tensor theory of gravitation. Rao et al. [10] have discussed LRS Bianchi type-I dark energy cosmological model in the BransDicke theory of gravitation. Rao et al. [11] have discussed Bianchi type-II, -VIII, and -IX dark energy cosmological models in the Saez-Ballester theory of gravitation. Recently, Rao et al. [12] have obtained perfect fluid dark energy cosmological models in the Saez-Ballester and general theory of gravitation.

Recently, there has been considerable interest in cosmological models with dark energy in general relativity because of the fact that our universe is currently undergoing an accelerated expansion which has been confirmed by a host of observations, such as type Ia supernovae (Reiss et al. [13]; Perlmutter et al. [14]; and Tegmark et al. [15]). Based on these observations, cosmologists have accepted the idea of dark energy, which is a fluid with negative presence making up around $70 \%$ of the present universe energy content to be responsible for this acceleration due to repulsive gravitation. Cosmologists have proposed many candidates for dark energy to fit the current observations such as cosmological constant, tachyon, quintessence, and phantom. Current studies to extract the properties of a dark energy component of the universe from observational data focus on the determination of its equation of state $w(t)$, which is the ratio of the dark energy's pressure to its energy density $w(t)=p / \rho$, which is not necessarily constant. The methods for restoration of the quantity $w(t)$ from experimental data have been developed (Sahni and Starobinsky [16]), and an analysis of the experimental data has been conducted to determine this parameter as a function of cosmological time (Sahni et al. [17]). The simplest dark energy candidate is the vacuum energy ( $w=$ -1 ), which is mathematically equivalent to the cosmological 
constant $(\Lambda)$. The other conventional alternatives, which can be described by minimally coupled scalar fields, are quintessence $(w>-1)$, phantom energy $(w<-1)$, and quintom (that can cross from phantom region to quintessence region as evolved) and have time dependent EoS parameter. Due to lack of observational evidence in making a distinction between constant and variable $w$, usually the equation of state parameter is considered as a constant (Kujat et al. [18]; Bartelmann et al. [19]) with phase wise value $-1,0,+1 / 3$, and +1 for vacuum fluid, dust fluid, radiation, and stiff dominated universe, respectively. But in general, $w$ is a function of time or redshift (Jimenez [20]; Das et al. [21]). Ray et al. [22], A. K. Yadav and L. Yadav [23], Kumar [24], and Pradhan et al. [25] are some of the authors who have investigated dark energy models in general relativity with variable EoS parameters in different contexts. Yadav and Saha [26] have obtained an LRS Bianchi-I anisotropic cosmological model with dominance of dark energy.

In this paper, we will study LRS Bianchi type-I dark energy cosmological models in the Nordtvedt [1] general scalar tensor theory with the help of a special case proposed by Schwinger [27], that is, $3+2 \omega(\phi)=(1 / \lambda \phi)$, where $\lambda$ is a constant.

\section{Metric and Field Equations}

We consider the LRS Bianchi type-I metric in the following form:

$$
d s^{2}=d t^{2}-A^{2} d x^{2}-B^{2}\left(d y^{2}+d z^{2}\right)
$$

where $A$ and $B$ are functions of cosmic time $t$ only.

The field equations of general scalar tensor theory proposed by Nordtvedt (using geometrized units with $c=1$, $G=1)$ are

$$
\begin{aligned}
R_{i j}- & \frac{1}{2} g_{i j} R \\
= & -8 \pi \phi^{-1} T_{i j}-\omega \phi^{-2}\left(\phi,_{i} \phi,_{j}-\frac{1}{2} g_{i j} \phi,_{k} \phi^{, k}\right) \\
& -\phi^{-1}\left(\phi_{i ; j}-g_{i j} \square \phi\right), \\
\square \phi & =\phi_{; k}^{, k}=\frac{8 \pi T}{3+2 \omega}-\frac{1}{(3+2 \omega)} \frac{d \omega}{d \phi} \phi_{,_{i}} \phi^{, i},
\end{aligned}
$$

where $R_{i j}$ is the Ricci tensor, $R$ is the curvature invariant, $T_{i j}$ is the stress energy of the matter, and comma and semicolon denote partial and covariant differentiation, respectively.

Also, we have

$$
T_{; j}^{i j}=0
$$

which is a consequence of the field equations (2).
The energy momentum tensor components of the fluid can be written in anisotropic diagonal form as

$$
T_{j}^{i}=\operatorname{diag}\left[T_{0}^{0}, T_{1}^{1}, T_{2}^{2}, T_{3}^{3}\right]
$$

We can parameterize the components of the energy momentum tensor as follows:

$$
\begin{aligned}
T_{j}^{i} & =\operatorname{diag}\left[\rho,-p_{x},-p_{y},-p_{z}\right] \\
& =\operatorname{diag}\left[1,-w_{x},-w_{y},-w_{z}\right] \rho \\
& =\operatorname{diag}[1,-w,-(w+\gamma),-(w+\gamma)] \rho,
\end{aligned}
$$

where $\rho$ is the energy density of the fluid. $p_{x}, p_{y}$, and $p_{z}$ are the pressures, $w_{x}, w_{y}$, and $w_{z}$ are the directional equation of state (EoS) parameters of the fluid along $x-, y$-, and $z$ axes, respectively, and $w(t)=(p / \rho)$ is the deviation-free EoS parameter of the fluid.

Here, we have parameterized the deviation from isotropy by setting $w_{x}=w$. Also $\gamma$ is the skewness parameter, which is a deviation from $w$ along $y$ - and $z$-axes. The parameters $w, \gamma$ are not necessarily constants and can be functions of the cosmic time $t$.

Using commoving coordinates, the field equations (2)-(3) for the metric (1) with the help of (5) can be written as

$$
\begin{gathered}
2 \frac{\ddot{B}}{B}+\left(\frac{\dot{B}}{B}\right)^{2}+\frac{\omega}{2}\left(\frac{\dot{\phi}}{\phi}\right)^{2}+\frac{\ddot{\phi}}{\phi}+2 \frac{\dot{\phi}}{\phi}\left(\frac{\dot{B}}{B}\right)=-8 \pi \phi^{-1} w \rho \\
\left(\frac{\ddot{A}}{A}\right)+\left(\frac{\ddot{B}}{B}\right)+\left(\frac{\dot{A} \dot{B}}{A B}\right)+\frac{\omega}{2}\left(\frac{\dot{\phi}}{\phi}\right)^{2}+\frac{\ddot{\phi}}{\phi}+\frac{\dot{\phi}}{\phi}\left(\frac{\dot{A}}{A}+\frac{\dot{B}}{B}\right) \\
=-8 \pi \phi^{-1}(w+\gamma) \rho, \\
2\left(\frac{\dot{A} \dot{B}}{A B}\right)+\left(\frac{\dot{B}}{B}\right)^{2}-\frac{\omega}{2}\left(\frac{\dot{\phi}}{\phi}\right)^{2}+\frac{\dot{\phi}}{\phi}\left(\frac{\dot{A}}{A}+2 \frac{\dot{B}}{B}\right) \\
=8 \pi \phi^{-1} \rho, \\
(3+2 \omega)\left(\ddot{\phi}+\dot{\phi}\left(\frac{\dot{A}}{A}+2 \frac{\dot{B}}{B}\right)\right) \\
=8 \pi \rho(1-2 \gamma-3 w)-\dot{\phi}^{2} \frac{d \omega}{d \phi}, \\
\dot{\rho}+\frac{\dot{B}}{A}(w+1) \rho+2 \frac{\dot{B}}{B}(w+1+\gamma) \rho=0
\end{gathered}
$$

where the overhead $\operatorname{dot}(\cdot)$ denotes derivative with respect to the cosmic time $t$. 
By using the transformation $A=e^{\alpha}, B=e^{\beta}$, and $d t=$ $e^{\alpha+2 \beta} d T$, the field equations (6) can be written as

$$
\begin{gathered}
2 \beta^{\prime \prime}-2 \alpha^{\prime} \beta^{\prime}-\beta^{\prime 2}+\frac{\omega}{2} \frac{\phi^{\prime 2}}{\phi^{2}}+\frac{\phi^{\prime \prime}}{\phi}-\frac{\phi^{\prime}}{\phi} \alpha^{\prime}=\frac{-8 \pi}{\phi} w \rho e^{2(\alpha+2 \beta)} \\
\alpha^{\prime \prime}+\beta^{\prime \prime}-2 \alpha^{\prime} \beta^{\prime}-\beta^{\prime 2}+\frac{\omega}{2} \frac{\phi^{\prime 2}}{\phi^{2}}+\frac{\phi^{\prime \prime}}{\phi}-\frac{\phi^{\prime}}{\phi} \beta^{\prime} \\
=\frac{-8 \pi}{\phi}(w+\gamma) \rho e^{2(\alpha+2 \beta)} \\
2 \alpha^{\prime} \beta^{\prime}+\beta^{\prime 2}-\frac{\omega}{2} \frac{\phi^{\prime 2}}{\phi^{2}}+\frac{\phi^{\prime}}{\phi}\left(\alpha^{\prime}+2 \beta^{\prime}\right)=\frac{8 \pi}{\phi} \rho e^{2(\alpha+2 \beta)} \\
(3+2 \omega) \phi^{\prime \prime}+\phi^{\prime 2} \frac{d \omega}{d \phi}=8 \pi \rho e^{2(\alpha+2 \beta)}(1-2 \gamma-3 w) \\
\rho^{\prime}+\frac{A^{\prime}}{A}(w+1) \rho+2 \frac{B^{\prime}}{B}(w+1+\gamma) \rho=0
\end{gathered}
$$

where the overhead dash denotes derivative with respect to $T$.

\section{Dark Energy Cosmological Models in General Scalar Tensor Theory of Gravitation}

The field equations (7) to (10) are four independent equations with seven unknowns $A, B, \omega, \phi, \rho, w$, and $\gamma$. Here, we obtain dark energy cosmological model in Nordtvedt's general scalar tensor theory in a special case proposed by Schwinger [27] in the following form:

$$
3+2 \omega(\phi)=\frac{1}{\lambda \phi}, \quad \lambda=\text { constant }
$$

From (7) to (10), we get

$$
\begin{gathered}
(3+2 \omega) \phi^{\prime \prime}-2 \phi\left[\alpha^{\prime \prime}+2 \beta^{\prime \prime}-\beta^{\prime 2}-2 \alpha^{\prime} \beta^{\prime}\right] \\
-\omega \frac{\phi^{\prime 2}}{\phi}-3 \phi^{\prime \prime}+\phi^{\prime 2} \frac{d \omega}{d \phi}=0 .
\end{gathered}
$$

From (12) and (13), we get

$$
\begin{aligned}
\frac{1}{\lambda}\left[\frac{\phi^{\prime \prime}}{\phi}\right. & \left.-\frac{\phi^{\prime 2}}{\phi^{2}}\right]-2 \phi\left[\alpha^{\prime \prime}+2 \beta^{\prime \prime}-\beta^{\prime 2}-2 \alpha^{\prime} \beta^{\prime}\right] \\
& +\frac{3}{2} \frac{\phi^{\prime 2}}{\phi}-3 \phi^{\prime \prime}=0 .
\end{aligned}
$$

Then, let us construct a physically meaningful model by considering

$$
\begin{aligned}
& \phi=e^{r\left(k_{1} T+k_{2}\right)}, \\
& A=e^{m\left(k_{1} T+k_{2}\right)}, \\
& B=e^{n\left(k_{1} T+k_{2}\right)} .
\end{aligned}
$$

Equations (15) will satisfy (14) provided that the arbitrary constants $m, n$, and $r$ are related by $4 n^{2}+8 n m-3 r^{2}=0$. For various values of $m, n$, and $r$, we will get different cosmological models.

From (9), we get the energy density

$$
\begin{aligned}
8 \pi \rho= & e^{-2(m+2 n)\left(k_{1} T+k_{2}\right)} k_{1}^{2} \\
& \times\left(e^{r\left(k_{1} T+k_{2}\right)}\left[n^{2}+2 n m+\frac{3}{4} r^{2}+r(m+2 n)\right]-\frac{1}{4 \lambda} r^{2}\right) .
\end{aligned}
$$

From (7), we get the EoS parameter

$$
w=\frac{\left(e^{r\left(k_{1} T+k_{2}\right)}\left[n^{2}+2 n m-(1 / 4) r^{2}+r m\right]-(1 / 4 \lambda) r^{2}\right)}{\left(e^{r\left(k_{1} T+k_{2}\right)}\left[n^{2}+2 n m+(3 / 4) r^{2}+r(m+2 n)\right]-(1 / 4 \lambda) r^{2}\right)} .
$$

From (7) and (8), we get the skewness parameter

$$
\gamma=\frac{r(n-m) e^{r\left(k_{1} T+k_{2}\right)}}{\left(e^{r\left(k_{1} T+k_{2}\right)}\left[n^{2}+2 n m+(3 / 4) r^{2}+r(m+2 n)\right]-(1 / 4 \lambda) r^{2}\right)} .
$$

Then, the metric (1) can be written in the following form:

$$
\begin{aligned}
d s^{2}= & e^{2(m+2 n)\left(k_{1} T+k_{2}\right)} d T^{2}-e^{2 m\left(k_{1} T+k_{2}\right)} d x^{2} \\
& -e^{2 n\left(k_{1} T+k_{2}\right)}\left(d y^{2}+d z^{2}\right)
\end{aligned}
$$

Thus, the metric (19) together with (16) to (18) constitutes LRS Bianchi type-I dark energy cosmological models in general scalar tensor theory of gravitation. Since $m, n$, and $r$ are arbitrary constants, for different values of $m, n$, and $r$, we will get different cosmological models. But in this paper, we will present the following anisotropic as well as isotropic cosmological models.

3.1. Anisotropic Dark Energy Cosmological Model in General Scalar Tensor Theory of Gravitation. Spatially homogeneous cosmological models play an important role in attempts to understand the structure and properties of the space of all cosmological solutions of Einstein field equations. Moreover, from the theoretical point of view, anisotropic universe has a greater generality than isotropic models. Although the present day universe is satisfactorily described by homogeneous and isotropic models given by the FriedmannRobertson-Walker (FRW) space-time, as we know the universe in a smaller scale is neither homogeneous nor isotropic nor do we expect the universe in its early stages to have these properties. In fact, to get a physically realistic description of the universe, one has to consider inhomogeneous models. In this case, the solutions of Einstein's field equations become more complicated or may be impossible. Therefore, many theoretical cosmologists are trying to use the spatially homogeneous and anisotropic Bianchi type models instead of inhomogeneous models. These types of space-times present a "middle way" between FRW models and inhomogeneous 
and anisotropic universes and hence play an important role in modern cosmology. get

If $m=-(1 / 2), n=(3 / 2)$, and $r=1$, from (15) to (18), we

$$
\begin{aligned}
& A=e^{-(1 / 2)\left(k_{1} T+k_{2}\right)}, \\
& B=e^{(3 / 2)\left(k_{1} T+k_{2}\right)}, \\
& \phi=e^{\left(k_{1} T+k_{2}\right)}, \\
& \rho=\frac{k_{1}^{2}\left[4 e^{\left(k_{1} T+k_{2}\right)}-(1 / 4 \lambda)\right]}{8 \pi e^{5\left(k_{1} T+k_{2}\right)}}, \\
& w=\frac{1}{1-16 \lambda e^{\left(k_{1} T+k_{2}\right)}}, \\
& \gamma=\frac{1}{2\left(1-(1 / 16 \lambda) e^{-\left(k_{1} T+k_{2}\right)}\right)} .
\end{aligned}
$$

Then, the model (19) can be written as

$$
\begin{gathered}
d s^{2}=e^{5\left(k_{1} T+k_{2}\right)} d T^{2}-e^{-\left(k_{1} T+k_{2}\right)} d x^{2} \\
+e^{3\left(k_{1} T+k_{2}\right)}\left(d y^{2}+d z^{2}\right) .
\end{gathered}
$$

Thus, the metric (21) together with $\phi, \rho, w$, and $\gamma$, as given above, constitutes LRS Bianchi type-I anisotropic dark energy cosmological model in general scalar tensor theory of gravitation.

3.2. Isotropic Dark Energy Cosmological Model in General Scalar Tensor Theory of Gravitation. If $m=n=1$ and $r=2$, from (15) to (18), we get

$$
\begin{gathered}
A=B=e^{\left(k_{1} T+k_{2}\right)}, \\
\phi=e^{2\left(k_{1} T+k_{2}\right)}, \\
\rho=\frac{k_{1}^{2}\left[12 e^{2\left(k_{1} T+k_{2}\right)}-(1 / \lambda)\right]}{8 \pi e^{6\left(k_{1} T+k_{2}\right)}}, \\
w=\frac{\left[4 e^{2\left(k_{1} T+k_{2}\right)}-(1 / \lambda)\right]}{\left[12 e^{2\left(k_{1} T+k_{2}\right)}-(1 / \lambda)\right]} \\
\gamma=0 .
\end{gathered}
$$

Then, the model (19) can be written as

$$
d s^{2}=e^{6\left(k_{1} T+k_{2}\right)} d T^{2}-e^{2\left(k_{1} T+k_{2}\right)}\left[d x^{2}+d y^{2}+d z^{2}\right] .
$$

Thus, the metric (23) together with $\phi, \rho, w$, and $\gamma$ as given above constitutes LRS Bianchi type-I dark energy cosmological model in the isotropic form in general scalar tensor theory of gravitation.

\section{Some Important Features of the Models}

4.1. Anisotropic Dark Energy Cosmological Model. The volume element of the model (21) is given by

$$
V=(-g)^{1 / 2}=e^{(5 / 2)\left(k_{1} T+k_{2}\right)} .
$$

Mean Hubble's parameter $H$ is given by

$$
H=\frac{1}{3}\left(H_{1}+2 H_{2}\right)=\frac{5 k_{1}}{6} .
$$

The deceleration parameter $q$ is given by

$$
q=-3 \theta^{-2}\left(\theta_{, i} u^{i}+\frac{1}{3} \theta^{2}\right)=-1 .
$$

Average anisotropy parameter $A_{m}$ is given by

$$
A_{m}=\frac{1}{3} \sum_{i=1}^{3}\left(\frac{\Delta H_{i}}{H}\right)^{2}=\frac{32}{25} \text {. }
$$

The expansion scalar $\theta$ is given by

$$
\theta=u_{, i}^{i}=\frac{5 k_{1}}{2} .
$$

The shear scalar is $\sigma^{2}$ defined as $\sigma^{2}=(3 / 2) A_{m} H^{2}$,

$$
\sigma^{2}=\frac{4}{3} k_{1}^{2}
$$

The overall density parameter $\Omega$ is given by

$$
\Omega=\frac{\rho}{3 H^{2}}=\frac{12}{25 e^{5\left(k_{1} T+k_{2}\right)}}\left(4 e^{\left(k_{1} T+k_{2}\right)}-\frac{1}{4 \lambda}\right) .
$$

The tensor of rotation $w_{i j}=u_{i, j}-u_{j, i}$ is identically zero, and hence, this universe is nonrotational.

4.2. Isotropic Dark Energy Cosmological Model. The volume element of the model (23) is given by

$$
V=(-g)^{1 / 2}=e^{3\left(k_{1} T+k_{2}\right)} .
$$

Mean Hubble's parameter $H$ is given by

$$
H=\frac{1}{3}\left(H_{1}+2 H_{2}\right)=k_{1} \text {. }
$$

The deceleration parameter $q$ is given by

$$
q=-3 \theta^{-2}\left(\theta_{, i} u^{i}+\frac{1}{3} \theta^{2}\right)=-1 .
$$

Average anisotropy parameter $A_{m}$ is given by

$$
A_{m}=\frac{1}{3} \sum_{i=1}^{3}\left(\frac{\Delta H_{i}}{H}\right)^{2}=0 .
$$

The expansion scalar $\theta$ is given by

$$
\theta=u_{, i}^{i}=3 k_{1} .
$$

The shear scalar is $\sigma^{2}$ defined as $\sigma^{2}=(3 / 2) A_{m} H^{2}$,

$$
\sigma^{2}=0 \text {. }
$$

The overall density parameter $\Omega$ is given by

$$
\Omega=\frac{\rho}{3 H^{2}}=\frac{1}{24 \pi e^{6\left(k_{1} T+k_{2}\right)}}\left(12 e^{2\left(k_{1} T+k_{2}\right)}-\frac{1}{\lambda}\right) .
$$

The tensor of rotation $w_{i j}=u_{i, j}-u_{j, i}$ is identically zero, and hence, this universe is nonrotational. 


\section{Conclusions}

In this paper, we have presented a spatially homogeneous LRS Bianchi type-I anisotropic as well as isotropic dark energy cosmological models in the Nordtvedt [1] general scalar tensor theory of gravitation with the help of a special case proposed by Schwinger [27]. For both the models, the spatial volume is constant at $T=-k_{2} / k_{1}$ and increases exponentially with time. This shows that at the initial epoch, the universe starts with constant volume and expands exponentially approaching infinite volume. It is observed that the model (19) is free from singularities. For both the models, the expansion scalar $\theta$ exhibits the constant value. This shows that the universe expands homogeneously. We observe that the shear scalar $\sigma$ and the Hubble parameter $H$ are also constants for both the models. From (21), we can observe that the energy density and the EoS parameter will become zero as $T \rightarrow$ $\infty$, while skewness parameter is constant. From (23), we can observe the energy density and skewness parameter will become zero as $T \rightarrow \infty$, while the EoS parameter is constant. For all the previous models, the overall density parameter vanishes as $T \rightarrow \infty$. Also, the deceleration parameter that appears with negative sign implies accelerating expansion of the universe as one can expect for exponential volumetric expansion, which is consistent with the present day observations. By recent observations of type Ia supernovae (SN Ia), Perlmutter et al. [14] and Riess et al. ([13, 28, 29]) proved that the decelerating parameter of the universe is in the range $-1 \leq q \leq 0$, and the present day universe is undergoing accelerated expansion. For the model (21), we can observe that $A_{m} \neq 0$ which indicates that the model is anisotropic and represents the early stages of the universe. Recent experiments show that there is a certain amount of anisotropy in the universe. Hence, anisotropic space-times are important. For the model (23), we can see that $A_{m}=0$, which indicates that the model is isotropic and represents the present stage of the universe. Thus, the anisotropic as well as isotropic dark energy cosmological models presented here are expanding, nonrotating, and accelerating in the standard way.

\section{Acknowledgment}

The second author (D. Neelima) is grateful to the Department of Science and Technology (DST), New Delhi, India, for providing INSPIRE fellowship.

\section{References}

[1] K. Nordtvedt Jr., "Post-Newtonian metric for a general class of scalar-tensor gravitational theories and observational consequences," The Astrophysical Journal, vol. 161, pp. 1059-1067, 1970.

[2] B. M. Barker, "General scalar-tensor theory of gravity with constant G," Astrophysical Journal, vol. 219, no. 1, pp. 5-11, 1978.

[3] V. A. Ruban and A. M. Finkelstein, "Generalization of the TaubKazner cosmological metric in the scalar-tensor gravitation theory," Lettere Al Nuovo Cimento Series 2, vol. 5, no. 3, pp. 289293, 1972.

[4] A. Banerjee and N. O. Santos, "Homogeneous radiation-filled Universe in general scalar tensor theory," Journal of Physics A, vol. 14, no. 10, article 2829, 1981.
[5] A. Banerjee and N. O. Santos, "Homogeneous cosmological model in general scalar-tensor theory," Physical Review D, vol. 23, pp. 2111-2115, 1981.

[6] K. Shanti and V. U. M. Rao, "Conformally flat static space-time in the general scalar-tensor theory of gravitation "' Astrophysics and Space Science, vol. 162, no. 1, pp. 163-165, 1989.

[7] K. Shanti and V. U. M. Rao, "Bianchi type-VI0 cosmological model in the general scalar-tensor theory of gravitation," Astrophysics and Space Science, vol. 172, no. 1, pp. 83-88, 1990.

[8] V. U. M. Rao and G. Sreedevi Kumari, "A cosmological model with negative constant deceleration parameter in a general scalar-tensor theory of gravitation," International Journal of Theoretical Physics, vol. 51, no. 1, pp. 311-315, 2012.

[9] V. U. M. Rao, G. S. Kumari, and B. J. M. Rao, "Kaluza-Klein radiating model in a general scalar-tensor theory," Astrophysics and Space Science, vol. 337, no. 2, pp. 795-798, 2012.

[10] V. U. M. Rao, M. Vijaya Santhi, T. Vinutha, and G. Sreedevi Kumari, "LRS Bianchi type-I dark energy cosmological model in Brans-Dicke theory of gravitation," International Journal of Theoretical Physics, vol. 51, no. 10, pp. 3303-3310, 2012.

[11] V. U. M. Rao, K. V. S. Sireesha, and P. Suneetha, "Bianchi type-II, VIII and IX dark energy cosmological models in saez-ballester theory of gravitation," The African Review of Physics, vol. 7, p. 0054, 2012.

[12] V. U. M. Rao, K. V. S. Sireesha, and D. Neelima, "Bianchi type II, VIII, and IX perfect fluid dark energy cosmological models in Saez-Ballester and general theory of gravitation," ISRN Astronomy and Astrophysics, vol. 2013, Article ID 924834, 11 pages, 2013.

[13] A. G. Reiss, A. V. Filippenko, P. Challis et al., "Observational evidence from supernovae for an accelerating universe and a cosmological constant," The Astrophysical Journal, vol. 116, article 1009, 1998.

[14] S. Perlmutter, G. Aldering, G. Goldhaber et al., "Measurements of $\Omega$ and $\Lambda$ from 42 High-Redshift Supernovae," The Astrophysical Journal, vol. 517, no. 2, article 565, 1999.

[15] M. Tegmark, M. R. Blanton, M. A. Strauss et al., "The threedimensional power spectrum of galaxies from the sloan digital sky survey," The Astrophysical Journal, vol. 606, no. 2, article 702, 2004.

[16] V. Sahni and A. Starobinsky, "Reconstructing dark energy," International Journal of Modern Physics D, vol. 15, no. 12, pp. 2105-2132, 2006.

[17] V. Sahni, A. Shafielooa, and A. Starobinsky, "Two new diagnostics of dark energy," Physical Review D, vol. 78, Article ID 103502, 11 pages, 2008.

[18] J. Kujat, A. M. Linn, R. J. Scherrer, and D. H. Weinberg, "Prospects for determining the equation of state of the dark energy: what can be learned from multiple observables?" The Astrophysical Journal, vol. 572, no. 1, 2002.

[19] M. Bartelmann, K. Dolagb, F. Perrotta et al., "Evolution of darkmatter haloes in a variety of dark-energy cosmologies," New Astronomy Reviews, vol. 49, no. 2-6, pp. 199-203, 2005.

[20] R. Jimenez, "The value of the equation of state of dark energy," New Astronomy Reviews, vol. 47, no. 8-10, pp. 761-767, 2003.

[21] A. Das, S. Gupta, T. D. Saini, and S. Kar, "Cosmology with decaying tachyon matter," Physical Review D, vol. 72, no. 4, Article ID 043528, 6 pages, 2005.

[22] S. Ray, F. Rahaman, U. Mukhopadhyay, and R. Sarkar, "Variable equation of state for generalized dark energy model," International Journal of Theoretical Physics, vol. 50, no. 9, pp. 26872696, 2010. 
[23] A. K. Yadav and L. Yadav, "Bianchi type III anisotropic dark energy models with constant deceleration parameter," International Journal of Theoretical Physics, vol. 50, no. 1, pp. 218-227, 2010.

[24] S. Kumar, "Some FRW models of accelerating universe with dark energy," Astrophysics and Space Science, vol. 332, no. 2, pp. 449-454, 2010.

[25] A. Pradhan, H. Amirhashchi, and B. Saha, "Bianchi typeI anisotropic dark energy model with constant deceleration parameter," International Journal of Theoretical Physics, vol. 50, no. 9, pp. 2923-2938, 2011.

[26] A. K. Yadav and B. Saha, "LRS Bianchi-I anisotropic cosmological model with dominance of dark energy," Astrophysics and Space Science, vol. 337, no. 2, pp. 759-765, 2012.

[27] J. Schwinger, Particles, Sources and Fields, Addison-Wesley, Reading, Mass, USA, 1970.

[28] A. G. Riess, R. P. Kirshner, B. P. Schmidt et al., "BVRI light curves for 22 type Ia supernovae," The Astronomical Journal, vol. 117, no. 2, article 707, 1999.

[29] A. G. Riess, L.-G. Strolger, J. Tonry et al., “Type Ia supernova discoveries at $z>1$ from the hubble space telescope: evidence for past deceleration and constraints on dark energy evolution," The Astrophysical Journal, vol. 607, no. 2, p. 665, 2004. 

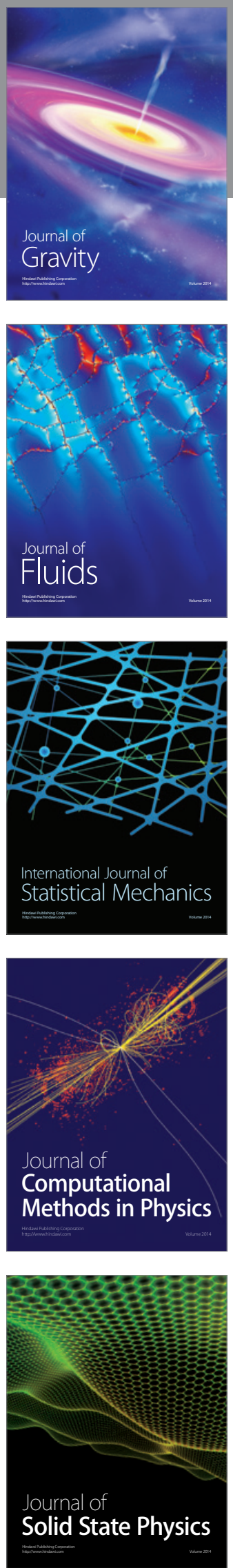

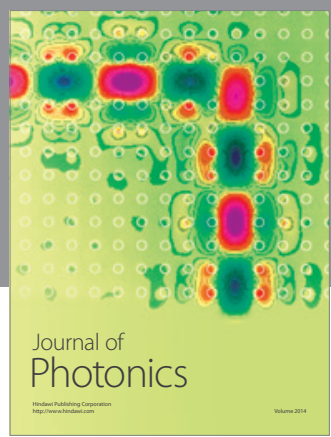

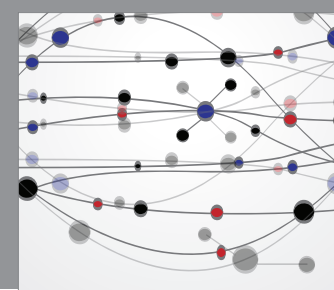

The Scientific World Journal

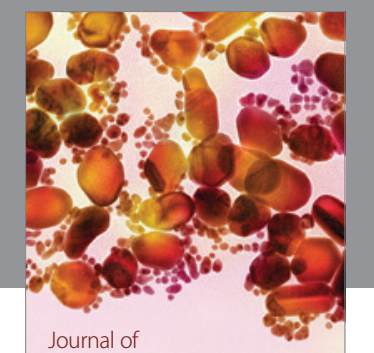

Soft Matter
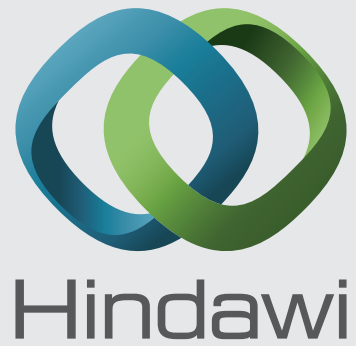

Submit your manuscripts at

http://www.hindawi.com
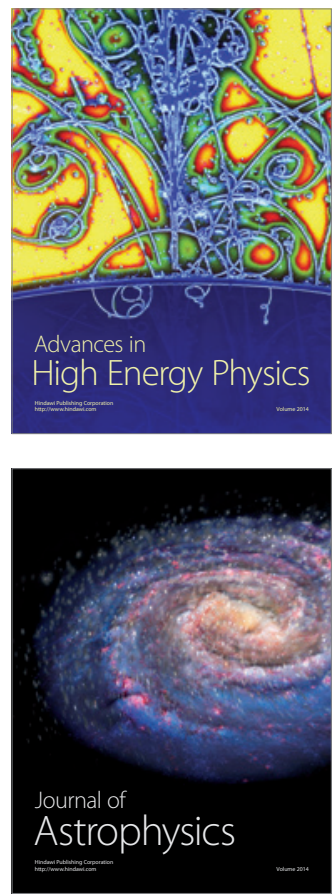
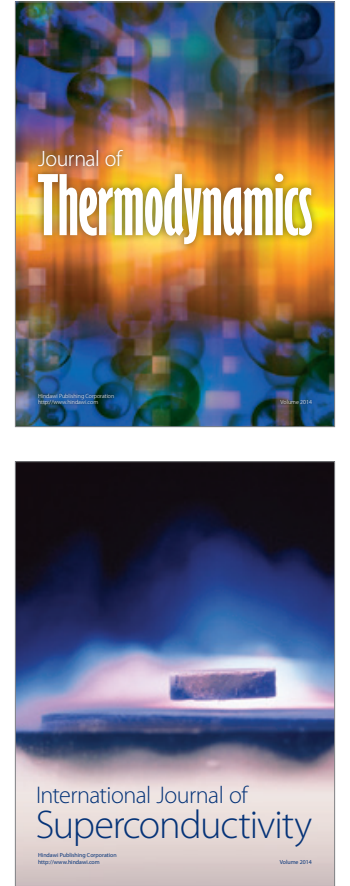
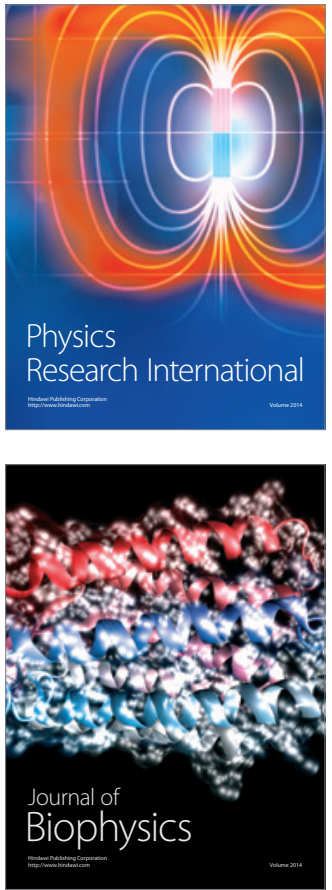
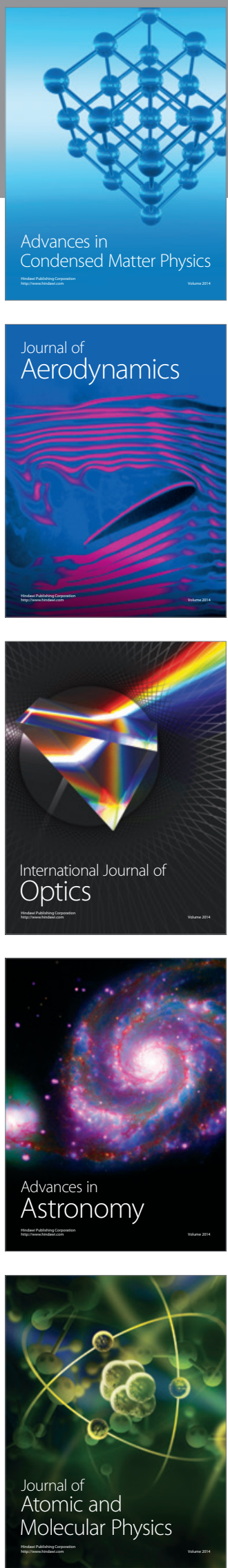